\title{
Josephson junction with magnetic-field tunable ground state
}

\author{
E. Goldobin, ${ }^{1}$ D. Koelle, ${ }^{1}$ R. Kleiner,${ }^{1}$ and R. G. Mints ${ }^{2}$ \\ ${ }^{1}$ Physikalisches Institut and Center for Collective Quantum Phenomena in LISA ${ }^{+}$, \\ Universität Tübingen, Auf der Morgenstelle 14, D-72076 Tübingen, Germany \\ ${ }^{2}$ The Raymond and Beverly Sackler School of Physics and Astronomy, Tel Aviv University, Tel Aviv 69978, Israel
}

(Dated: November 24, 2018)

\begin{abstract}
We consider an asymmetric $0-\pi$ Josephson junction consisting of 0 and $\pi$ regions of different lengths $L_{0}$ and $L_{\pi}$. As predicted earlier this system can be described by an effective sine-Gordon equation for the spatially averaged phase $\psi$ so that the effective current-phase relation of this system includes a negative second harmonic $\propto \sin (2 \psi)$. If its amplitude is large enough, the ground state of the junction is doubly degenerate $\psi= \pm \varphi$, where $\varphi$ depends on the amplitudes of the first and second harmonics. We study the behavior of such a junction in an applied magnetic field $H$ and demonstrate that $H$ induces an additional term $\propto H \cos \psi$ in the effective current-phase relation. This results in a non-trivial ground state tunable by magnetic field. The dependence of the critical current on $H$ allows for revealing the ground state experimentally.
\end{abstract}

Josephson junctions (JJs) with a phase shift of $\pi$ in the ground state [1] attracted a lot of interest in the recent years [2 7]. These JJs can be used as onchip phase batteries for biasing various classical [8] and quantum [9] circuits. This allows for removing external bias lines and reducing decoherence. Currently, it is possible to fabricate simultaneously both 0 and $\pi$ JJs using various technologies such as superconductorferromagnet heterostructures 10] or JJs based on d-wave superconductors 11 13.

It would be remarkable to have a JJ (a phase battery) providing an arbitrary phase shift $\varphi$, rather than just 0 or $\pi$. Long arrays of $0-\pi-0-\pi-\ldots$ JJs with short segments were suggested as systems, where the $\varphi \mathrm{JJ}$ can be realized [14 16]. Each of the segments are assumed to have a standard current-phase relation (CPR) $j_{s}= \pm j_{0} \sin \phi$. The phase $\phi$ in these systems can be written as a sum of two terms $\phi(x)=\psi+\xi(x) \sin \psi$, where $\psi$ is a constant spatially averaged phase and $|\xi(x)| \ll 1$ is alternating on a scale of the order of the segment's length. The effective CPR then reads 14 16.

$$
j_{s}=j_{1} \sin (\psi)+j_{2} \sin (2 \psi),
$$

where $j_{1}=\left\langle j_{c}(x)\right\rangle$ is the spatially averaged critical current density and $j_{2}<0$ is the amplitude of the effective second harmonic. We note that special stochastic distributions of facets may also lead to $j_{2}>0$ [17]. The value of $j_{2}$ depends on parameters of the junctions. For $j_{2}<-j_{1} / 2$ the ground state of the system is doubly degenerate with $\psi= \pm \varphi$, where

$$
\varphi=\arccos \left[-j_{1} /\left(2 j_{2}\right)\right] .
$$

This $\varphi$ JJ[16] is the generalization of a $\pi$ JJ and can provide an arbitrary phase bias $0<\varphi<\pi$. These JJs have unusual physical properties [18], e.g., non quantized Josephson vortices that were predicted [14] and observed experimentally 15$]$.

The simplest system, which is suitable for the realization of a "short" $\varphi$ JJ, is an asymmetric (i.e., $L_{0} \neq L_{\pi}$ )
0- $\pi$ JJ with relatively short segments of length $L_{0}, L_{\pi} \lesssim$ $\lambda_{J}$, where $\lambda_{J} \propto 1 / \sqrt{j_{0}}$ is the Josephson length. Such a system can be considered as one period of an infinitely long $0-\pi-0-\pi-\ldots$ chain. The best method to reveal the $\varphi$ JJ experimentally is to include it in a superconducting loop (thus forming a SQUID) and to measure the spontaneously generated flux. Another option is to study these JJs in a magnetic field $H$. It is worth mentioning that there is an apparent contradiction here. On one hand, the usual linear phase ansatz $\phi(x)=H x+\phi_{0}$ in asymmetric $0-\pi$ JJ results in a critical current $I_{c}(H)$ with a cusplike minimum at $H=0$, see Eq. (11) in Ref. 11 as well as Refs. 19, 20. This $I_{c}(H)$ should be mirror symmetric with respect to the $I_{c}$ axis, i.e., $I_{c}(-H)=I_{c}(+H)$. On the other hand, the presence of the second harmonic in the CPR (1) and the linear phase ansatz for $\psi$ leads to a Fraunhofer-like $I_{c}(H)$ with the maximum at $H=0$ [18]. Moreover, when $\left|j_{2}\right|>0.5 j_{1}$ there are two critical currents $I_{c \pm}(H)$, corresponding to depinning of the phase from different potential wells, but both of the $I_{c \pm}(H)$ dependences have the main maximum at $H=0$. 18

In this paper we study the asymmetric $0-\pi$ JJ in a magnetic field in detail. We demonstrate that the amplitude of the second harmonic $j_{2}$ in Eq. (1) depends only on the JJ parameters, while the magnetic field $H$ induces an additional term $\propto H \cos \psi$. Further, we predict the dependence of $I_{c}(H)$ at small magnetic fields.

Consider an asymmetric JJ shown in Fig. 1. The dependence $j_{c}(x)$ is given by

$$
\begin{array}{ll}
j_{c}(x)=-j_{0}, & \text { if }-L_{\pi}<x<0 ; \\
j_{c}(x)=+j_{0}, & \text { if } 0<x<L_{0}
\end{array}
$$

The behavior of the phase $\phi(x)$ is defined by the static sine-Gordon equation

$$
\phi^{\prime \prime}(x)-j_{c}(x) \sin [\phi(x)]=-\gamma,
$$

where the coordinate $x$ and lengths $L_{0, \pi}$ are normalized in the usual way to $\lambda_{J}$ and $\gamma=j / j_{0}$ is the normalized 


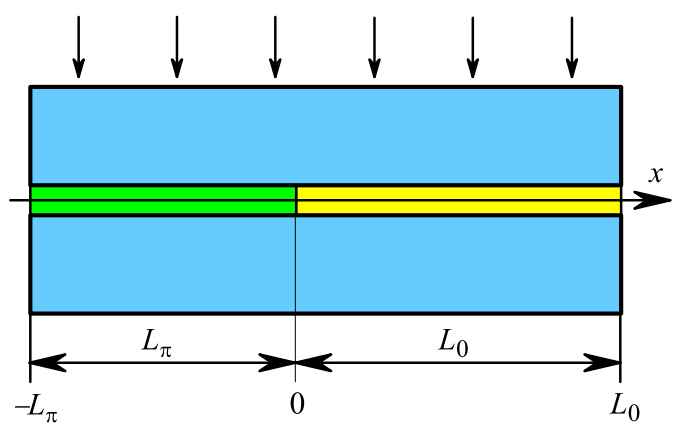

FIG. 1: (Color online) Geometry of the $0-\pi$ JJ.

bias current density. $j_{c}(x)$ below is also normalized to $j_{0}$. Next, we write 14]

$$
j_{c}(x)=\left\langle j_{c}\right\rangle[1+g(x)],
$$

where

$$
\left\langle j_{c}\right\rangle=\frac{1}{L_{0}+L_{\pi}} \int_{-L_{\pi}}^{+L_{0}} j_{c}(x) d x=j_{0} \frac{L_{0}-L_{\pi}}{L_{0}+L_{\pi}}
$$

is the average value of the critical current density and

$$
g(x)=\left\{\begin{array}{l}
g_{\pi}=\frac{-2 L_{0}}{L_{0}-L_{\pi}}, \text { for } x<0 ; \\
g_{0}=\frac{+2 L_{\pi}}{L_{0}-L_{\pi}}, \text { for } x>0,
\end{array}\right.
$$

is the deviation from the average value $(\langle g(x)\rangle=0)$.

Thus, we rewrite Eq. (4) in terms of $g(x)$ and arrive to

$$
\phi^{\prime \prime}-\left\langle j_{c}\right\rangle[1+g(x)] \sin \phi=-\gamma .
$$

In what follows we treat short JJs $\left(L_{0}, L_{\pi} \lesssim 1\right)$. Therefore, the solution $\phi(x)$ can be sought in the form 14]

$$
\phi(x)=\psi+\xi(x) \sin \psi,
$$

where $\psi$ is a constant 26] and $\xi(x)$ describes small variations of the phase around $\psi$, i.e., $|\xi(x)| \ll 1,\langle\xi(x)\rangle=0$. Substituting the ansatz (9) into Eq. (8) and expanding to the first order in $\xi(x)$ we get

$$
\xi^{\prime \prime} \sin \psi-\left\langle j_{c}\right\rangle[1+g(x)][1+\xi(x) \cos \psi] \sin \psi=-\gamma .
$$

Equation (10) has two types of terms: the constant ones and varying ones. Note, that the term $g(x) \xi(x)$ has both the constant (average) part $\langle g(x) \xi(x)\rangle$ and the deviation from the average $g(x) \xi(x)-\langle g(x) \xi(x)\rangle$. Thus, from Eq. (10) the relation for the constant terms reads

$$
\gamma=\left\langle j_{c}\right\rangle[\sin \psi+\langle g(x) \xi(x)\rangle \sin \psi \cos \psi] .
$$

The equation for $\xi(x)$, recalling definition (5), is

$$
\xi^{\prime \prime}-j_{c}(x) \cos \psi \xi(x)=\left\langle j_{c}\right\rangle[g(x)-\langle g \xi\rangle \cos \psi],
$$

It turns out that both terms $\propto \cos \psi$ have an extremely weak influence on the results. Therefore, for the sake of simplicity, we omit them right away to arrive to

$$
\xi^{\prime \prime}=\left\langle j_{c}\right\rangle g(x)
$$

Solutions of this equation include four constants to be determined from the matching conditions at $x=0$

$$
\xi_{\pi}(0)=\xi_{0}(0) ; \quad \xi_{\pi}^{\prime}(0)=\xi_{0}^{\prime}(0),
$$

and boundary conditions at $x=-L_{\pi}$ and $x=L_{0}$

$$
\xi_{\pi}^{\prime}\left(-L_{\pi}\right) \sin \psi=h ; \quad \xi_{0}^{\prime}\left(L_{0}\right) \sin \psi=h,
$$

where $h=2 H / H_{c 1}$ is the normalized applied magnetic field, $H_{c 1}=\Phi_{0} /\left(\pi \lambda_{J} \Lambda\right)$ is the penetration field and $\Lambda \approx$ $2 \lambda_{L}$ is the effective magnetic thickness of the JJ. Thus, we arrive to the expression for $\xi(x)$ and can calculate

$$
\langle g \xi\rangle=\Gamma_{0}+\Gamma_{h} \frac{h}{\sin \psi},
$$

where the coefficients

$$
\Gamma_{0}=-\frac{4}{3} \frac{L_{0}^{2} L_{\pi}^{2}}{L_{0}^{2}-L_{\pi}^{2}} ; \quad \Gamma_{h}=\frac{L_{0} L_{\pi}}{L_{0}-L_{\pi}} .
$$

Thus, Eq. (11) gives the effective CPR of the JJ

$$
\gamma=\left\langle j_{c}\right\rangle\left[\sin \psi+\Gamma_{h} h \cos \psi+\frac{\Gamma_{0}}{2} \sin (2 \psi)\right] .
$$

The result is remarkable - as in earlier works 14 16 one gets the second harmonic $\sin (2 \psi)$ with the negative amplitude of $\Gamma_{0} / 2$. In addition, the magnetic field results in a $\Gamma_{h} h \cos \psi$ term which additionally modifies the CPR and is tunable by magnetic field. Fig. 2 (a) shows the effective CPRs $\gamma(\psi)$ of a 0- $\pi$ JJ for several different values of magnetic field $h$. At $h=0$ we might have a doubly degenerate ground state $\psi= \pm \varphi$ which, upon application of $h$, transforms into a single one.

The Josephson energy corresponding to CPR (18) is

$$
U(\psi)=\left\langle j_{c}\right\rangle\left[1-\cos \psi+\Gamma_{h} h \sin \psi+\frac{\Gamma_{0}}{2} \sin ^{2} \psi\right] .
$$

The plots of $U(\psi)$ for the same set of $h$ values is shown in Fig. 2(b). Following the evolution of the curves at different $h$ one can see how the doubly degenerate ground state transforms into a single one. Moreover, one can see that at non-zero $h$ the potential energy $U(\psi)$ lacks reflection symmetry and therefore can be used to build Josephson phase ratchets 21, 22].

The effective CPR (18) at $h=0$ allows to calculate the domain of existence of nontrivial solutions, which is defined as $\left|\Gamma_{0}\right|>1$ [18]. This results in

$$
L_{\pi} \geq L_{0} \sqrt{\frac{3}{4 L_{0}^{2}+3}} ; \quad L_{0} \geq L_{\pi} \sqrt{\frac{3}{4 L_{\pi}^{2}+3}} .
$$




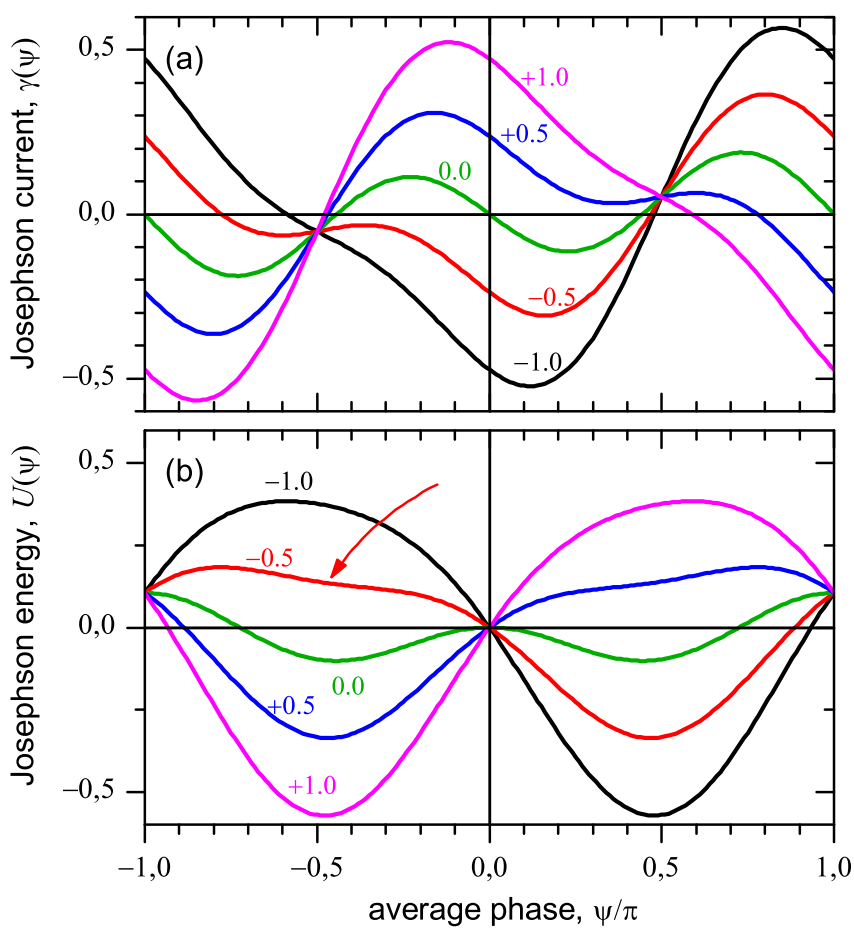

FIG. 2: (Color online) (a) effective CPR $\gamma(\psi)$ and (b) effective Josephson energy $U(\psi)$ of a $0-\pi$ JJ with $L_{0}=1, L_{\pi}=0.9$ for several different values of magnetic field $h$ given next to each curve. The arrow in (b) points to a local energy minimum, which appears at certain values of bias current.

Since we have made certain approximations, namely used Eq. (13) instead of Eq. (12), it is worth to compare the domain (20) with the exact result [23], which reads

$$
\begin{aligned}
& L_{\pi} \geq \arctan \left(\tanh \left(L_{0}\right)\right) ; \\
& L_{0} \geq \arctan \left(\tanh \left(L_{\pi}\right)\right),
\end{aligned}
$$

for our case $j_{c}^{0}=j_{c}^{\pi}$. Both boundaries, the exact one given by Eq. (21) and the approximate one given by Eq. (20) are shown in Fig. 3. It is seen that our approximation works extremely well not only for small $L_{0}$ and $L_{\pi}$, but also in the limits $L_{0}, L_{\pi} \rightarrow \infty$, where it deviates from the asymptotic value by only $\sim 10 \%$.

In the JJ with the CPR (18) and energy (19), one may have several stable static solutions and several critical (depinning) currents corresponding to the escape of the phase from the relevant energy minimum. To find the critical currents for a given magnetic field, we look for extrema of the CPR (18) with respect to $\psi$. We arrive to

$$
\cos \psi-\Gamma_{h} h \sin \psi-\Gamma_{0}\left(2 \cos ^{2} \psi-1\right)=0 .
$$

This problem can be reduced to a solution of a fourth order polynomial, i.e., all solutions of Eq. (22) can be found numerically. As a result we obtain up to four relevant roots and up to four corresponding critical currents.

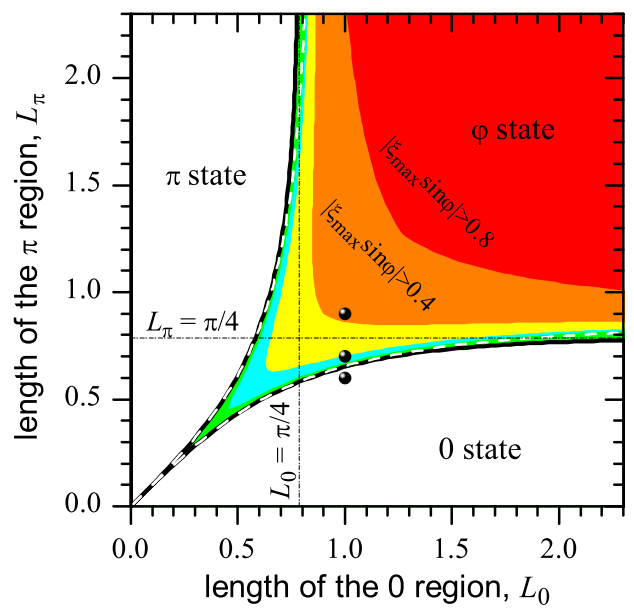

FIG. 3: The domain of existence of a $\varphi$ state $(h=0)$ on the $L_{0}, L_{\pi}$ plane. The exact boundary (21) (continuous lines) and our approximation (20) (dashed lines) agree very well. The $\varphi$ domain has regions of different colors from green (approximation $|\xi(x) \sin \psi| \ll 1$ works well) to red (approximation is invalid). The boundaries correspond to $\left|\xi_{\max } \sin \varphi\right|=0.1,0.2,0.4,0.8$. The dots indicate parameters used for discussion of Fig. 4 Vertical and horizontal dash-dotted lines show asymptotic behavior of exact boundary (21).

Several examples of $\gamma_{c}(h)$ dependences are shown in Fig. 4. One can see that for the parameter set (a), which corresponds to a state deep in the $\varphi$ region at $h=0$, see Fig. 3, one observes a characteristic rotated diamond-like shape with four critical currents in total for $|h| \lesssim 0.6$. The two branches that meet at $h \approx+0.6(-0.6)$ correspond to the escape from the left (right) potential well in Fig. 2(b). The upper (lower) branch corresponds to the escape in the right (left) direction. At $|h| \approx 0.6$ the branches meet indicating the disappearance of the corresponding local energy minimum. The two critical currents $\gamma_{c \pm}$ (in each direction) observed at $h=0$ coinside with $\gamma_{ \pm}$obtained in ref. 18. One may be able to observe the lower one experimentally in a system with low enough damping using a special sweep sequence 18]. For some values of $h$ the smaller minimum can even be absent at $\gamma=0$ [see the arrow Fig. 2(b)] and appear at larger $|\gamma|$ [the vertical dotted line corresponding to $h=0.5$ in Fig. [(a)]. The small energy minimum appears for $0.03<\gamma<0.06$, which also be seen in Fig. 2(b). Another distinct feature, which might be measurable in experiment, is the shift of the main minimum to $h= \pm 0.11$ in Fig. 4(a). This shift is the evidence of a non-trivial phase state in the junction. Finally, at large $|h|$ the upper branches of $\gamma_{c}(h)$ approach the asymptotic lines

$$
\gamma_{c}^{\text {as }}(h) \approx \pm\left\langle j_{c}\right\rangle \Gamma_{h} h,
$$

which are also shown in Fig. 4 We remind that our analysis is valid only for small values of $|h|$ and does not 

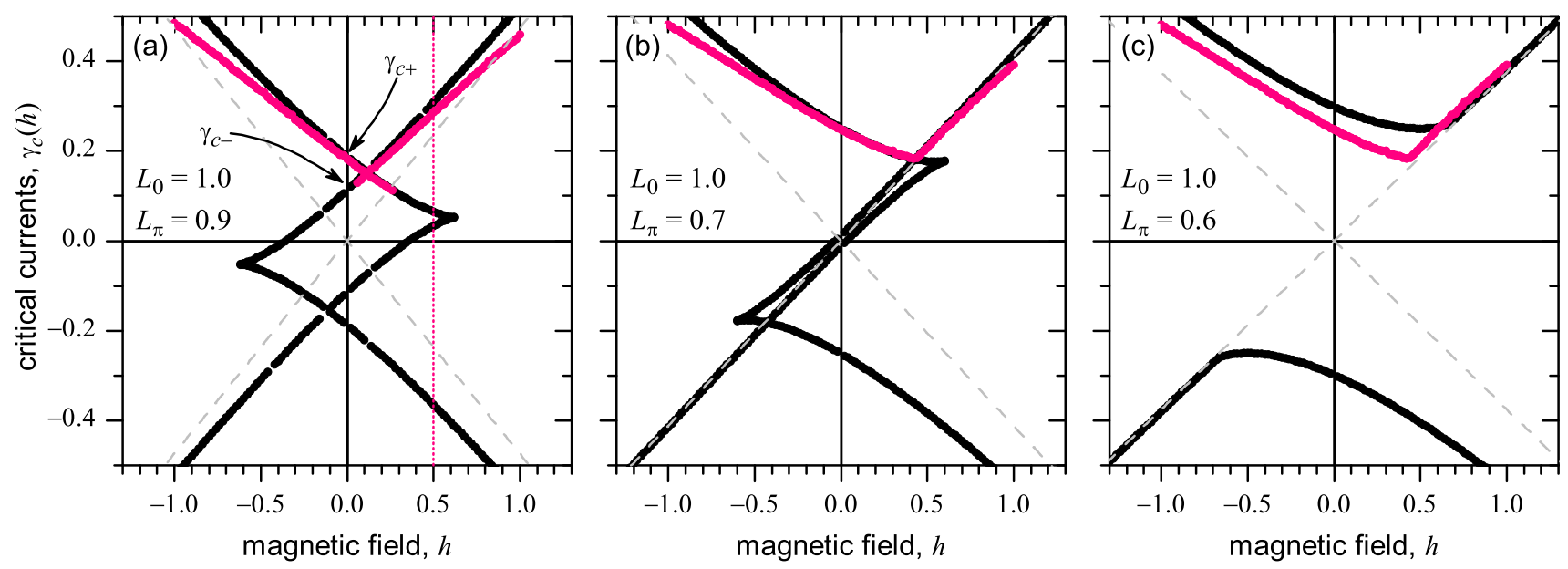

FIG. 4: (Color online) The critical current $\gamma_{c}(h)$ for $0-\pi$ JJs with different segment lengths. Dashed lines show the asymptotic behavior (23) for large $|h|$. Gray (pink) solid lines show the results of direct numerical simulations.

reproduce the global $\gamma_{c}(h)$ features, presented elsewhere.

When the $0-\pi$ JJ gets more asymmetric, see Fig. 4(b) and $(\mathrm{c})$, the lower $\gamma_{c}(h)$ diamond-shaped domain becomes thinner and finally collapses. The point of collapse corresponds to the crossing of the $\varphi$-domain boundary in Fig. 3. Looking at $\gamma_{c}(h)$ one can see that the main cusplike minimum shifts with asymmetry which can be used to extract the asymmetry from experimental data.

We also have performed numerical simulations of the $\gamma_{c}(h)$ dependence using StкJJ software 24. The above analytical results coincide with the results of direct numerical simulation, shown by pink (gray) in Fig. 4, with good accuracy. This is especially so at $h \ll 1$ when the approximation $|\xi(x) \sin \psi| \ll 1$, used to derive (10), is valid.

To summarize, we have shown that the effective CPR (18) includes the term $\propto H \cos \psi$, which allows to tune the ground state. Thus, our system is a hybrid between $\varphi$ [16] and $\varphi_{0}$ [25] JJs introduced earlier. The corresponding Josephson energy profile can be made asymmetric allowing to build Josephson phase ratchets. We have clarified how the $I_{c}(H)$ dependence of asymmetric $0-\pi$ JJ looks like for low magnetic field. It does have a minimum, but the minimum is shifted to some $\pm h_{\min }$ for positive and negative bias, respectively, so that the $I_{c}(H)$ curve is point symmetric.

We acknowledge financial support by the German Israeli Foundation (Grant No. G-967-126.14/2007).

[1] L. N. Bulaevskii, et al., JETP Lett. 25, 290 (1977).

[2] J. J. A. Baselmans, et al., Nature 397, 43 (1999).

[3] V. V. Ryazanov, et al., Phys. Rev. Lett. 86, 2427 (2001).

[4] T. Kontos, et al., Phys. Rev. Lett. 89, 137007 (2002).
[5] M. Weides, et al., Appl. Phys. Lett. 89, 122511 (pages 3) (2006), cond-mat/0604097.

[6] J. A. van Dam, et al., Nature (London) 442, 667 (2006), ISSN 0028-0836.

[7] A. Gumann, et al., Appl. Phys. Lett. 91, 192502 (pages 3) (2007).

[8] T. Ortlepp, et al., Science 312, 1495 (2006).

[9] A. K. Feofanov, et al., Nat. Phys. 6, 593 (2010), ISSN $1745-2473$.

[10] M. Weides, et al., Phys. Rev. Lett. 97, 247001 (pages 4) (2006), cond-mat/0605656.

[11] D. J. Van Harlingen, Rev. Mod. Phys. 67, 515 (1995).

[12] C. C. Tsuei et al., Rev. Mod. Phys. 72, 969 (2000).

[13] H.-J. H. Smilde, et al., Phys. Rev. Lett. 88, 057004 (2002).

[14] R. G. Mints, Phys. Rev. B 57, R3221 (1998).

[15] R. G. Mints, et al., Phys. Rev. Lett. 89, 067004 (2002).

[16] A. Buzdin et al., Phys. Rev. B 67, 220504(R) (pages 4) (2003), cond-mat/0305142.

[17] E. Il'ichev, et al., Phys. Rev. B 59, 11502 (1999).

[18] E. Goldobin, et al., Phys. Rev. B 76, 224523 (pages 13) (2007), 0708.2624.

[19] M. Kemmler, et al., Phys. Rev. B 81, 054522 (2010).

[20] M. Weides, et al., Supercond. Sci. Technol. 23, 095007 (2010).

[21] P. Reimann, Phys. Rep. 361, 57 (2002), condmat/0010237.

[22] P. Hänggi et al., Rev. Mod. Phys. 81, 387 (2009).

[23] L. N. Bulaevskii, et al., Solid State Commun. 25, 1053 (1978).

[24] E. Goldobin (2003), URL http://www.geocities.com/SiliconValley/Heights/7318/StkJJ.h

[25] A. Buzdin, Phys. Rev. Lett. 101, 107005 (2008).

[26] In Ref. $14 \psi$ is a slowly changing function of $x$. Here, we have only one period (two segments) taken separately and assume $\psi=$ const. The assumption $\psi=$ const makes the linear phase ansatz $\psi=h x+\psi_{0}$ used in Ref. 18 invalid. Instead, $h$ enters in the boundary conditions (15) for $\xi(x)$. 